\title{
CISTO MESENTÉRICO: RELATO DE CASO E REVISÃO DE LITERATURA
}

\author{
Mesenteric cyst: case report and literature review
}

\author{
Carlos MAGNO-JUNIOR, Carlos Augusto Marques BATISTA, Gerson França LEITE, \\ Hélio Alves de LIMA, Joaquim Ferreira de PAULA, Monika P. KIM, Wilker Benedetti MENDES
}

\begin{abstract}
Correspondência:
Carlos Magno Jr, e-mail: loscarmagno@hotmail.com

Fonte de financiamento: não há

Conflito de interesses: não há

Recebido para publicação:

Aceito para publicação:
\end{abstract}

Trabalho realizado no Hospital Escola Luiz Gioseffi Jannuzzi, Valença, RJ, Brasil.

\section{INTRODUÇÃO}

Os cistos mesentéricos são tumores intra-

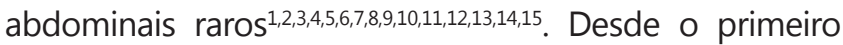
relato feito por Benevienae em 1507, aproximadamente 800-900 casos de cistos mesentéricos foram descritos na literatura ${ }^{1,3,7,14}$. Sua incidência pode variar conforme os diferentes trabalhos, de um em cada 27.000 a 250.000 admissões hospitalares ${ }^{1,7,15}$, ou então de 1:20.000 crianças e 1:100.000 adultos 9,12,14,15. A sua distribuição é igual em ambos os sexos ${ }^{1,5,7}$, predominando na fase adulta da vida1,4,11,12. A localização mais comum é no intestino delgado $1,7,9,14,15$. Normalmente são tumores assintomáticos ou com manifestações clínicas discretas e não específicas $1,2,3,5,6,7,8,9,10,12,12,13,14,15$. O diagnóstico é feito pela presença da massa abdominal, em investigações clínicas ou por quadro de abdome agudo, principalmente durante o ato cirúrgico 1,3,5,7,8,9,11,12,14,15. O tratamento de escolha é a enucleação do cisto, seja por laparotomia ${ }^{1,3,4,4,7,8,9,10,11,12,13,14,15}$ ou videolaparoscopia $2,3,6,9,11,12,13,14$.

O objetivo deste trabalho foi apresentar um único caso de cisto mesentérico operado no Serviço de Cirurgia Geral do Hospital Escola Luiz Gioseffi Jannuzzi e fazer revisão da literatura.

\section{RELATO DO CASO}

Mulher de 61 anos, branca, relatou que há aproximadamente um ano começou a apresentar quadro de dor abdominal de pequena intensidade em flanco direito e fossa ilíaca direita, sem outros sintomas associados. Há seis meses notou aparecimento de tumoração abdominal pouco dolorosa, móvel, de crescimento progressivo e localizada em flanco direito. Procurou ambulatório de ginecologia e ao exame apresentou massa abdominal em situação superior, sem pertencer a anexos, que estavam totalmente livres; ultrassonografia transvaginal evidenciou formação cística de 10,9 x 6,6 × 8,4 cm ocupando o flanco direito, sugerindo doença ovariana. Tomografia abdominal mostrou formação ovalar de aspecto cístico localizada no flanco direito, medindo $8,0 \mathrm{~cm}$ de diâmetro transverso, compatível com diagnóstico de cisto mesentérico (Figura 1A). Foi encaminhada ao ambulatório de cirurgia geral. Tinha história pregressa de hipertensão arterial; hipotireoidismo; cesareana e salpingectomia. Foi submetida à laparotomia mediana onde foi encontrado tumoração cística de paredes finas e lisas em mesentério de jejuno, de aproximadamente $12 \times 8 \mathrm{~cm}$ com ovários normais. O cisto foi totalmente retirado sem lesão de sua cápsula (Figura 1B).

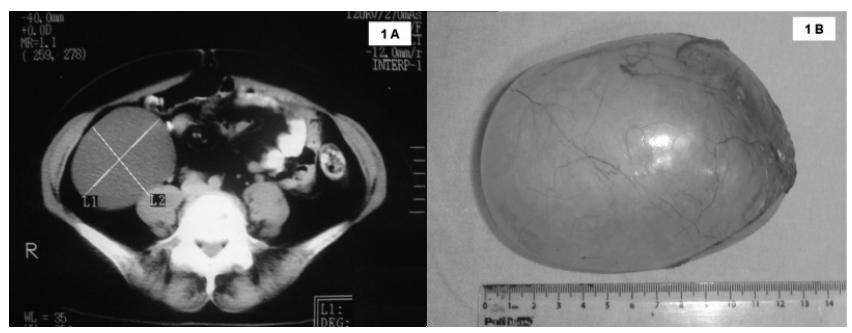

FIGURA 1 - A - Tomografia abdominal demonstrando lesão cística. B - Cisto mesentérico após enucleação

Evoluiu sem intercorrências, recebendo alta hospitalar no $2^{\circ}$ dia de pós-operatório. O laudo anatomopatológico mostrou formação cística transparente, medindo $12 \times 8 \times 6 \mathrm{~cm}$, com líquido incolor no seu interior; compatível com cisto mesentérico simples e sem sinais de malignidade. 0 acompanhamento tardio transcorreu sem complicações.

\section{DISCUSSÃO}

"Os cistos mesentéricos estão entre as raridades cirúrgicas", esta frase escrita por Berkeley Moynihan em 1887, reflete a opinião da maioria dos autores que escrevem sobre o assunto ${ }^{1,15}$. Tem baixa incidência e escassez de publicações ${ }^{1}$.

Pode ser definido como qualquer lesão cística localizada no mesentério ${ }^{1}$. É a mais rara das lesões císticas do abdome, geralmente é única, podendo 
seu tamanho variar de alguns centímetros até 30 centímetros de diâmetro ${ }^{7,14}$.

Desde o primeiro relato feito pelo anatomista italiano Benevienae em 1507, aproximadamente 800900 casos de cistos mesentéricos foram descritos na literatura ${ }^{1,3,7,14}$. A primeira excisão foi realizada por Tillaux em $1880^{1}$.

Sua incidência pode variar de um em cada 27.000 a 250.000 admissões hospitalares ${ }^{1,7,15}$, ou de 1:20.000 crianças e 1:100.000 adultos 9,12,15. Sua distribuição quanto ao gênero não é unânime, com relatos de incidência igual em ambos os sexos ${ }^{1,5,7}$, predominância no sexo feminino ${ }^{7,15}$ e outros trabalhos mostrando o sexo masculino como o mais acometido ${ }^{4,12}$. Predomina na fase adulta da vida ${ }^{1,4,11,12}$. Podem acometer o mesentério do duodeno ao reto ${ }^{1,7}$. A localização mais comum (60\%) é no intestino delgado $1,7,9,14,15$, preponderantemente na região do mesentério ileal ${ }^{5,6,12}$, podendo ainda ser encontrado no mesoapêndice ${ }^{3}$ eno mesocolon ${ }^{1,2,3,4,7,8,14}$. Pode ser originado de tecido linfático, vascular, nervoso e conjuntivo ${ }^{7,12,15}$. Embora exista certa contradição ${ }^{1}$, os cistos mesentéricos podem ser classificados como provenientes dos canais linfáticos, de divertículos entéricos, da crista urogenital ou de seus derivados, de infecções circunscritas ou de origem maligna ${ }^{1,7,9,12,14,15}$. O tipo histológico mais comum é o cisto quiloso ou linfático ${ }^{8,15}$. São histologicamente malignos em aproximadamente $3 \%$ dos $\operatorname{casos}^{14}$.

Normalmente são tumores assintomáticos ou com manifestações clínicas discretas e não específicas s, $2,3,5,6,7,8,9,10,12,13,14,15$. Quando sintomáticos podem se apresentar com dor abdominal vaga, náuseas e vômitos, constipação, diarréia e massa abdominal13,5,7,7,14. Geralmente, trata-se de uma massa abdominal compressível com grande mobilidade no sentido transversal e ao redor de um eixo central - sinal de Tillaux ${ }^{1}$.

O diagnóstico costuma ser feito pela presença da massa abdominal, durante investigação de doenças abdominais ou outras doenças, em achados acidentais de laparotomias ou então por quadro de abdome agudo, principalmente durante o ato cirúrgico ${ }^{1,3,5,7,7,9,10,11,12,13,14,15}$.

Exames de imagem, como ultrassonografia, tomografia e ressonância magnética são os que propiciam melhor acuidade diagnóstica, mostrando a massa cística uni ou multiloculada, procurando estabelecer a natureza, a localização e o tamanho da lesão, como também a espessura de suas paredes ${ }^{1,2,3,3,5,7,8,9,11,12,14,15}$.

O diagnóstico diferencial dos cistos mesentéricos pode ser feito, nas formas crônicas, com hidronefrose, lipoma de mesentério, tumores de intestino, cisto pancreático, cisto ovariano, cisto renal, cisto esplênico, cisto de adrenal e aneurisma de aorta ${ }^{1,7,9,14}$ e nas formas agudas com apendicite aguda, volvo, invaginação intestinal e obstrução intestinal ${ }^{1,11}$.

As complicações encontradas, que levam a quadro de abdome agudo, podem ser infecção espontânea, ruptura, infecção peritonial, hemorragia, torção do cisto, herniação para bolsa escrotal, volvo, isquemia intestinal, e obstrução intestinal $4,5,7,9,10,11,14$. Alguns autores citam a transformação maligna como complicação da doença9.

Uma vez diagnosticado, todo cisto mesentérico deve ser ressecado, a fim de evitar suas complicações ${ }^{1}$. O tratamento de escolha é a enucleação do cisto, seja por laparotomia ${ }^{1,3,4,5,7,8,9,10,11,12,14,15}$ ou videolaparoscopia $1,2,3,6,7,9,11,12,13,14,15$. Marsupialização, drenagem interna, aspiração do cisto e excisão parcial com cauterização são outras formas de tratamento descritas na literatura ${ }^{1,7}$.

A mortalidade operatória varia em função do tipo de tratamento efetivado, condição do paciente, estrutura de suporte pós-operatório e localização do cisto. Atualmente, com o aprimoramento tecnológico e o surgimento das modernas medidas é esperada mortalidade para a enucleação de $0 \%$ e de $3-5 \%$ com ressecção intestinal ${ }^{1,7}$.

A recidiva é rara e o tratamento pode ser considerado curativo ${ }^{2,11,15}$.

\section{REFERÊNCIAS}

1. Alves Júnior A, Maximiano L, Pires PWA, Birolini D. Cisto mesentérico. Rev.Hosp. Clin. Fac. Med. Univ. São Paulo 1994; 49(5):31-6.

2. Asoglu O, Igci A, Karanlik $H$, Parlak $M$, Kecer $M$, Ozmen V, Muslumanoglu M. Laparoscopic treatment of mesenteric cyst. Surg Endosc 2003; 17(5):832.

3. Ciulla A, Tomasello G, Castronovo G, Genova G, Maiorana AM. Laparoscopic treatment of mesenteric cysts. Report of two cases. Ann Ital Chir 2008; 79 (1): 63-5.

4. Desai N, Desai D, Ghag G, Waghela J, Rao RV, Sawant P. Giant mesenteric cyst of abdomen herniating into scrotum. Indian J Gastroenterol 2004; 23 (2): 74-5.

5. Ekçi B, Ayan F, Gürses B. Ruptured mesenteric cyst: a rare presentation after trauma. Ulus Travma Acil Cerrahi Derg 2007; 13 (1):74-7.

6. Festugato $\mathrm{M}$, Doncatto $\mathrm{EL}$, Fontana R. Ressecção de cisto mesentérico por videolaparoscopia: apresentação de caso. Rev. Cient. AMECS 1999; 8 (2):83-6.

7. Hassan M, Dobrilovic N, Korelitz J. Large gastric mesenteric cyst: case report and literature review. Am Surg 2005; 71 (7):571-3.

8. Miliaras S, Trygonis S, Papandoniou A, Kalamaras S, Trygonis C, Kiskinis D. Mesenteric cyst of the descending colon: report of a case. Acta Chir Belg 2006; 106 (6): 714-6.

9. Montiel-Jarquín JA, Romero-Morales LE, Enríquez-Adalid RM, Mendoza-García VA, Reyes-Páramo P, López-Colomo A. Mesoappendix cyst. A case report. Rev Med Inst Mex Seguro Soc An. 2008; 46 (5): 551-2.

10. Ozdogan M. Acute abdomen caused by a ruptured spontaneously infected mesenteric cyst. Turk J Gastroenterol 2004; 15 (2):120-1.

11. Pisano G, Erdas E, Parodo G, Martinasco L, Pomata M, Daniele GM. Acute abdomen duo to rupture of mesenteric cysts. Observations on a clinical case and review of the literature. Minerva Chir 2004; 59 (4): 405-11.

12. Roll S, Azevedo JLMC, Campos F, Gorski W, Abdala R, Azevedo OC. Ressecção de cisto mesentérico do tipo mesotelial por acesso videolaparoscópico. Rev. Col. Bras. Cir. 1996; 23 (5): 301-2.

13. Sahin DA, Akbulut G, Saykol V, San O, Tokyol C, Dilek ON. Laparoscopic enucleation of mesenteric cyst: a case report. Mt Sinai J Med 2006; 73 (7):1019-20.

14. Theodoridis TD, Zepiridis L, Athanatos D, Tzevelekis F, Kellartzis D, Bontis JN. Laparoscopic management of mesenteric cyst: a case report. Cases J 2009; 2: 132.

15. Yasojima EY, Cruz MMM, Valenta TON. Cisto mesentérico. Rev. Col. Bras. Cir. 2002; 29(3):177-8. 